\title{
An evolutionary and functional view of mammalian adipose tissue
}

\author{
BY CAROLINE M. POND \\ Department of Biology, The Open University, Milton Keynes MK7 6AA
}

The main function of adipose tissue is uptake, storage and controlled release of lipids; processes that involve large and rapid changes in tissue mass. The most efficient place for such a tissue is inside the abdomen near the centre of gravity and that is where most of the adipose tissue of amphibians and fast-moving reptiles such as lizards is found. The storage tissues of insects and other invertebrates are also intra-abdominal. In nearly all mammals except pinnipeds (seals) and certain small cetaceans (whales and dolphins), and in many birds, adipose tissue is partitioned into a dozen or more discrete depots that are widely distributed around the body. The tissue forms structurally intimate relations with skin, skeletal and cardiac muscle and various abdominal organs. Although very familiar to mammalian physiologists and biochemists, it is important to emphasize that, viewed in the context of the animal kingdom as a whole, the anatomy of adipose tissue in mammals and birds is unusual, and, in view of its primary function, illogical. There is no satisfactory explanation of why functionally, or when, in evolutionary time, this arrangement evolved.

It is becoming increasingly clear that total adiposity is determined more by appetite and energy expenditure than by the adipose tissue itself (Rössner \& Björntorp, 1989). However, although brain and endocrine mechanisms may determine the total abundance of the tissue, it is difficult to see how they could account for the relative sizes of different depots. The distribution of adipose tissue must be controlled, at least in part, by properties of the adipose tissue itself. Site-specific properties may, therefore, be among the features of adipose tissue that have the most direct bearing on obesity and animal production.

Until recently, white adipose tissue in mammals was simply described as 'widespread' (Ashwell, 1985) and its distribution was regarded as inconsistent and not amenable to functional or phylogenetic interpretation. It was regarded as a Cinderella tissue, filling in any spaces not occupied by other tissues. Curiously, the one exception to the notion that white adipose tissue 'has no gross anatomy' is the persistent belief that it accumulates between the skin and the superficial muscles because it is essential for thermal insulation. Despite its popularity, there is very little evidence that supports this theory but comparisons between arctic and temperate-zone species provide a rare opportunity to test it rigorously.

\section{SITE-SPECIFIC PROPERTIES OF ADIPOSE TISSUE}

Some site-specific properties of adipose tissue in laboratory rodents are summarized in Table 1. Each depot has a unique combination of properties under the conditions studied and so must make a specific contribution of energy homeostasis in the animal as a whole. This list is not exhaustive and we can confidently expect that further work will reveal more differences between depots which will help to elucidate their functions. The information presented shows that the intermuscular depots are usually the most 
metabolically active and responsive. The two categories of intra-abdominal adipose tissue have contrasting properties, some of which are shared with certain superficial and intermuscular depots. The two anterior superficial depots (shoulder and interscapular) have many properties in common with the intermuscular adipose tissue, while biochemical activity in the posterior superficial groin depot is relatively low. It is clear from Table 1 that the terms 'intra-abdominal' and 'superficial' describe only the depots' anatomical relations to other tissues and should not be taken to imply that the adipose tissue in these locations forms discrete categories with common physiological properties.

Adipocyte volume has long been assumed to be at least a predicator and probably a determinant of biochemical activity (Engfeldt \& Arner, 1988), but the information summarized in Table 1 shows that, when homologous depots in different species, or different depots in the same specimens are compared, mean adipocyte volume proves to be irrelevant as an indicator of metabolic activity. Intermuscular adipose tissue always has relatively high glycolytic capacity, whether it consists of large (as in the popliteal depot) or small (as in the neck intermuscular depot) adipocytes, or is derived from a lean or an obese specimen. Adipocyte volume is a useful marker for different depots, but this feature cannot explain the other site-specific properties. Although site-specific differences in adipocyte volume seem to be one of the most consistent features of adipose tissue in mammals, we cannot account for their origin, or suggest a function for them.

Identification of these differences leads to two important questions that relate the gross anatomy to the biochemistry. How do differences in the relative sizes of the depots affect the animal's metabolism as a whole? And, how far do the site-specific biochemical properties explain the anatomical relationships between the depots? It is becoming increasingly clear that the answers to these questions are likely to prove complicated. For example, attempts to account for anthropometric data and changes in adipocyte volume after weight reduction in terms of site-specific differences in lipoprotein lipase $(E C$ 3.1.1.34; LPL) activity and the action of noradrenaline and synthetic agonists on adipocytes in vitro have been remarkably unsuccessful (Mårin et al. 1990; Presta et al. 1990).

\section{THE RELATIVE MASSES OF THE DEPOTS}

It is now clear that the distribution of adipose tissue differs greatly between species, and to a lesser extent between individuals of the same species. Such differences are so consistent, and so resistant to perturbations such as pregnancy and large changes in total adiposity (Ashwell et al. 1986), that we must assume that they are physiologically adaptive. The relative masses of different depots is probably integrated with the diet, feeding and exercise habits or digestive physiology of the species but we have no idea how. Although it has been known for 40 years that atypical distribution of adipose tissue is associated with systemic diseases in humans, the metabolic consequences of deviant arrangements of adipose tissue have been little studied in any other species. Genetic manipulation of the distribution of adipose tissue in domestic livestock is now a real possibility but we have no idea what the long-term consequences might be for the health and energetic efficiency of the animals. It has so far proved impossible to manipulate substantially the relative sizes of the major depots in laboratory rodents by any means less drastic than surgical ablation, so comparative studies may be the most fruitful approach to the question of how the site-specific properties determine nutrient assimilation and energy balance in the whole animal. 


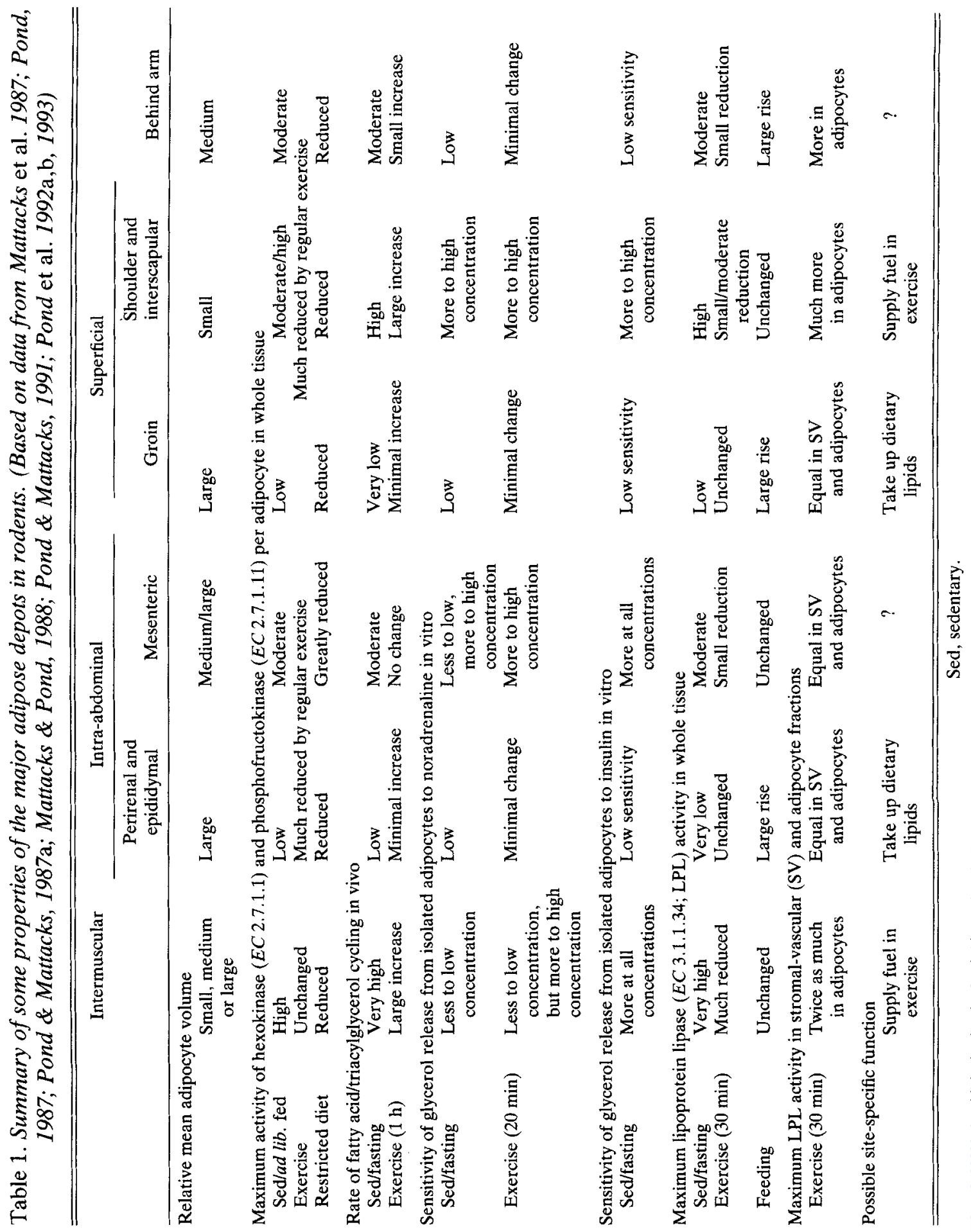


Of the properties listed in Table 1 , only those of the first two, adipocyte volume and maximum hexokinase ( $E C$ 2.7.1.1; HK) and phosphofructokinase ( $E C$ 2.7.1.11; PFK) activities, have been shown to occur in mammals other than rodents. The logistic difficulties of collecting numerous different samples simultaneously from large wild mammals are formidable, and effectively exclude studies on living cells or on enzymes that decay rapidly. Nevertheless, even such limited information is sufficient to make it clear that there is a definite organization of adipose tissue at the gross anatomical level that is common to all terrestrial mammals. The relative volume of adipocytes in different depots is constant (the absolute size depends on fatness and other factors) but the relative numbers of such adipocytes, and hence the relative masses of the depots, varies greatly between species, and to a lesser extent between individuals of the same species.

The intermuscular depots are always relatively small, usually $4-15 \%$ of the total dissectible adipose tissue. They are proportionately larger in lean individuals and, because they expand only slightly with increasing fatness, they represent only a small proportion of the total adipose tissue in obese specimens. The intermuscular depots have higher capacity for glucose utilization, take up more 2-deoxyglucose in vivo (Mattacks \& Pond, 1991), and have higher LPL activity (Pond et al. 1992b). Nonetheless, at least in guinea-pigs and hamsters, the intermuscular depots do not enlarge disproportionately as the animals fatten, even under conditions of minimal exercise: the extra lipid and glucose must be dissipated in some way, possibly by the high rate of fatty acid-triacylglycerol (FA/TAG) cycling (Mattacks \& Pond, 1988). The highest rate of FA/TAG cycling and of noradrenaline-stimulated lipolysis, and the lowest LPL activity in intermuscular adipose tissue occur following exercise, so the efficiency with which these processes match each other must depend at least in part on the magnitude and frequency of exercise.

The intra-abdominal depots also have distinctive properties. Those of the mesenteric depot could be related to its position near to the site of absorption from the gut and the fact that it is drained by the portal blood system. But if the depot does selectively take up newly absorbed lipids, this role cannot be indispensable because the depot is minimal in some insectivores, even obese specimens, and is virtually absent in many seals and small toothed whales. In general, larger species have proportionately less intra-abdominal adipose tissue, and more superficial, probably because the abdominal viscera are also proportionately smaller in larger mammals (Pond \& Ramsay, 1992). Massive hypertrophy of the intra-abdominal depots, while the superficial adipose tissue remains constant or is depleted, is common in humans (Enzi et al. 1986) but is very rarely seen in wild mammals, even in specimens that have been confined in captivity for many years.

The relative sizes of the superficial depots vary even more between species. The groin depots are usually relatively large, at least $15 \%$ of the total adipose tissue in most rodents and insectivores, but they are small in horses and hares, and in camels they account for only about $1 \%$ of the adipose tissue (Pond, 1986). The diet of equids, lagomorphs and camels is low in lipids, although quite large quantities of short-chain fatty acids are synthesized by bacterial digestion. In rodents (Mattacks et al. 1987; Pond et al. 1992b) and humans (Rebuffé-Scrive, 1987), the groin is the most metabolically inert of the storage depots, having low capacity for glucose uptake and (except after feeding) low LPL activity. In carnivores, these depots extend over the biceps femoris muscle of the leg towards the tail so that in large, obese specimens almost the whole of the posterior half of the body is encased in adipose tissue that consists of large adipocytes with low glycolytic capacity (Pond et al. 1992a). These depots may be specifically involved in the metabolism 
of dietary lipids, particularly those containing long-chain fatty acids, but this idea remains to be tested.

Adipose tissue in front and behind the forelimb ('shoulder' and 'arm') are also very variable, each accounting for one-quarter of the adipose tissue in the myomorph rodent Phodopus sungorus (dwarf hamster) but less than $5 \%$ in deer, and less than $10 \%$ in guinea-pigs. In contrast to these large differences between taxa, the ratio of the mass of posterior superficial adipose tissue (mostly groin and around the base of the tail) to that of anterior superficial adipose tissue (mostly around the forelimb and interscapular) is constant in species of similar fatness belonging to several different families of Carnivora (Pond \& Ramsay, 1992). Taken together, these observations suggest that the relative development of different superficial depots is integrated with the metabolism and homeostasis of the animal, but present knowledge is insufficient to explain how.

In general, the more massive depots are less metabolically active and less responsive than the smaller depots. Such differentials may be an efficient way of combining the storage of large quantities of lipid with rapid, accurate response to the small, routine requirements of meals and exercise. A high rate of FA/TAG cycling, and responsiveness to noradrenaline, equip the tissue to respond rapidly to changes in energy flux, but 'futile' cycles are energetically expensive to sustain continuously and generate significant quantities of heat (Newsholme, 1977). If these properties are confined to a few small depots, energy economy can be combined with responsiveness. The inescapable conclusion from such considerations is that the small, less frequently studied depots contribute much more to routine, day-to-day glucose and lipid homeostasis than the more massive depots. Many of the metabolic properties and responses of intermuscular adipose tissue to hormones such as noradrenaline, and the fact that in natural obesity the mass of such depots changes relatively little, are consistent with the hypothesis that it acts as a local energy source for adjacent muscles that share its blood supply, but such interactions have not been demonstrated directly.

One difficulty with such concepts is that in natural obesity, though not necessarily in artificial or pathological obesity, the more metabolically inert depots expand more than the intermuscular depots. At least in bears, disproportionate growth of the superficial depots is due almost entirely to the accumulation of additional adipocytes: the ratio of mean adipocyte volumes in internal and superficial depots is constant. It is not clear from the few biochemical properties that we have been able to measure how such selective accumulation arises. It has been suggested from observations on rats and humans that adipocyte enlargement stimulates proliferation or maturation of pre-adipocytes, or both (Sjöström \& Williams-Olsson, 1981; Björntorp et al. 1982). However, in bears, the depots with the highest glycolytic capacity, and, by homology with rodents, the highest LPL activity (Pond et al. 1992b), do not necessarily accumulate more adipocytes, and hence expand disproportionately, with increasing total fatness. If anything, the few data available suggest that the opposite is true (Pond et al. 1992a). So either the Björntorp et al. (1982) theory is flawed, or one of the differences between natural obesity in wild mammals and pathological or artificial obesity in humans and laboratory rodents is the creation of a causal link between enlargement and proliferation of adipocytes.

Like many large mammals, humans have several additional adipose depots that are minimal or absent in rodents. Unfortunately, such depots not only often become massive, they are also convenient for biopsies, so they are frequently chosen for study in preference to less accessible depots that are homologous to those of laboratory species 
(Frayn et al. 1989). Intermuscular adipose tissue is often abundant in humans, but almost nothing is known about its properties. The expansion of the one easily measured intermuscular depot, the part of the popliteal adipose mass that extends over the calf muscle, correlates very poorly with that of other depots of the same person (Garn et al. 1987; Johnston et al. 1988).

\section{SUPERFICIAL ADIPOSE TISSUE AND THERMAL INSULATION}

Wild arctic mammals provide the ideal opportunity to examine critically the theory that superficial adipose tissue has evolved as an adaptation to thermal insulation. The hypothesis is testable in two ways; first, the partitioning of adipose tissue between internal and superficial depots may be adapted to increase the contribution to thermal insulation. Second, the metabolism and chemical composition may be adapted to continual existence of a temperature gradient across the superficial adipose tissue.

The order Carnivora includes tropical, temperate and arctic species ranging in adult body mass from less than 1 to more than $500 \mathrm{~kg}$. At high latitudes, food supply is highly seasonal and often variable from year to year and place to place, so many arctic species are obese for at least part of the year. Most temperate-zone and tropical ecosystems are much more complex and the predators have several, often many, alternative sources of food that they can turn to when favoured prey become scarce. Long periods of fasting are rare so selection does not favour accumulating large quantities of fat except in the case of hibernating forms. Polar bears are top carnivores that feed almost exclusively on seals (mainly Phoca hispidus). Both numbers of seals and the ice conditions that favour predation vary with season and location, so bears frequently remain active for months without feeding during the summer, and rapidly become obese when food is plentiful in the winter and spring. Their ecological environment clearly accounts for the abundance of adipose tissue in this species, but we also wanted to know whether adaptive changes in the distribution of the tissue had evolved in response to the cold climate (Pond \& Ramsay, 1992). To isolate the effects of body mass on partitioning of adipose tissue, it was essential to compare specimens of similar body composition, which in practice meant captive or winter dormant carnivores. The regression lines on Fig. 1 are fitted to all the data except those from the polar bears, but the measurements on these large arctic carnivores nonetheless fall very close to the line. Such a comparison reveals that the partitioning of adipose tissue between superficial and intra-abdominal depots in polar bears is not significantly different from that which would be expected in temperate-zone and tropical carnivores of similar body mass. In other words, there is no evidence that the distribution of adipose tissue in this winter-active arctic species is adapted to the cold climate. The 'extra' adipose tissue goes on the outside in increasing quantities with greater fatness and larger body mass in all carnivores, regardless of their need for thermal insulation. The greatest accumulation is in the posterior superficial depots which have relatively low glycolytic capacity (Pond et al. 1992a), and by homology with laboratory species, probably low lipogenic and lipolytic activity as well. In such locations, the extra storage lipid may be biochemically as well as anatomically 'out of the way'.

Although comparative studies show that there has been no net shift in the partitioning of adipose tissue in arctic species, there is some evidence for biochemical adaptation to a thermal gradient across the tissue. Reindeer, Rangifer tarandus, particularly Rangifer tarandus platyrhynchus which has evolved to subspecies status on the Svalbard archipel- 


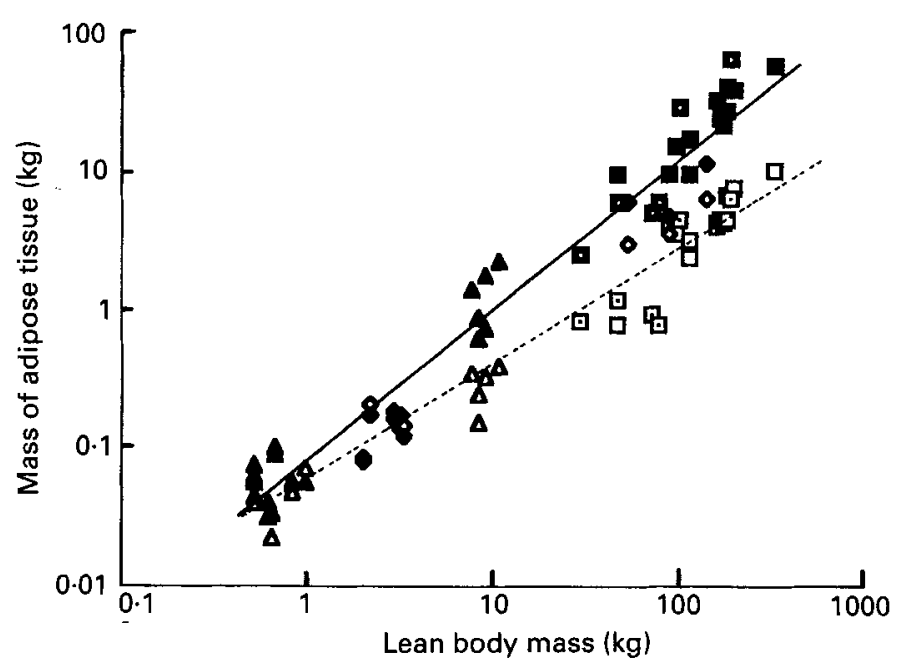

Fig. 1. The mass of all superficial $(\mathbf{\Delta}-\mathbf{\Lambda}, \mathbf{\square -} \mathbf{\square}, \quad-\quad, \mathbf{\square -})$ ) and all intra-abdominal $(\triangle--\triangle, \square--\square, \quad--\quad$, $\square--\square)$ adipose tissue as a function of lean body mass in forty-one adult and sub-adult Carnivora over $8.4 \%$ by weight dissectible adipose tissue. $\mathbf{\Delta}, \triangle$, Mustelidae (eight Mustela erminea, six Meles meles); , , Felidae (five Felis catus, one Panthera onca, one $P$. tigris, one $P$. leo), $\square, \square$, thirteen Ursus maritimus; $\square, \square$, six $U$. arctos. The regression lines shown are fitted to all data except those from $U$. maritimus. From Pond $\&$ Ramsay (1992).

ago in the absence of any natural predators, feed intensively and fatten rapidly during the brief arctic summer and autumn. As in carnivores, the superficial depots enlarge disproportionately and may become more than $100 \mathrm{~mm}$ thick over the rump and hind-quarters. The unsaturation index of the FA/TAG is slightly though consistently higher in samples taken from the outer side than from the inner side of five thick superficial depots (Pond et al. 1993). A similar trend was detected in superficial adipose tissue of polar bears, although it was less clearcut, because all the storage lipids contain a much higher proportion of unsaturated fatty acids than reindeer. These findings suggest that all the skin-side of adipose tissue is normally cooler than the inner side, as expected on the insulation hypothesis, and that the composition of the storage lipids is adapted to this situation. However, nothing is known of the physiological mechanisms that bring about these adaptive modifications in lipid distribution.

\section{SEX DIFFERENCES}

Sex differences in the distribution and abundance of adipose tissue, although conspicuous in humans (Enzi et al. 1986), are minimal in other primates (Pond \& Mattacks, 1987b; Laber-Laird et al. 1991). Several physiological differences between the sexes on a time-scale of minutes or hours have been described in rodents: for example, after $1 \mathrm{~h}$ of exercise, the rate of FA/TAG cycling in both intermuscular depots and the muscles themselves increased more in females, but the response of the superficial depots to this treatment was greater in males (Mattacks \& Pond, 1988). Female guinea-pigs run just as readily as males, but the decreases in LPL in intermuscular and certain superficial depots 


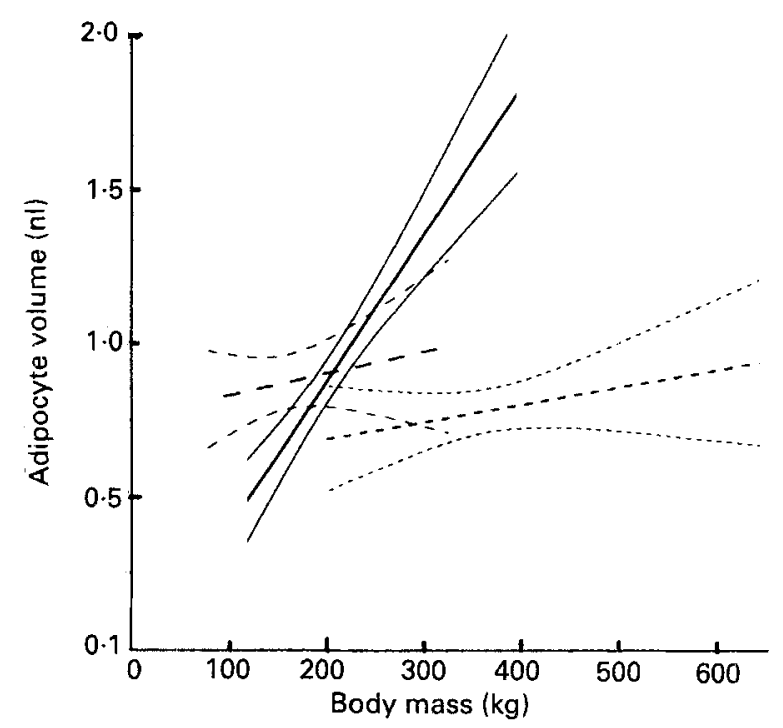

Fig. 2. The relationship between the mean adipocyte volume of a single biopsy taken from the hind leg near the tail and total body mass in wild polar bears (Ursus maritimus) caught in March-May and August-November in the Canadian Arctic. Regression lines are shown with $95 \%$ confidence intervals. $(-)$, Adult females $(n 176)$; $(---)$, adult males ( $n 112)$; $(---)$, juveniles ( $n$ 82, forty-nine males, thirty-three females). From Ramsay et al. (1992).

after 30 min of exercise were more consistent in males than in females (Pond et al. $1992 b$ ). However, on a time-scale of weeks, these properties apparently do not lead to substantial differences in distribution beyond those that arise from the fact that the epididymal depot is relatively large in male murid rodents (Pond et al. 1986; 1987).

The reproductive energetics and life-history strategy of female polar bears is quite different from that of males but the only detectable sex differences in the adipose tissue are in its micro-anatomy and metabolism, not the gross anatomy. Adipocytes enlarge much more in adult females (Fig. 2) than in adult males and such enlargement is accompanied by large increases in body mass (probably due mainly to growth of lean tissue) in males. These findings indicate that females fatten extensively without major changes in the mass of lean tissue. There is also evidence in dwarf hamsters for sex differences in the relative growth of fat and lean tissue and in the contributions of adipocytes enlargement and adipocyte proliferation to this process. On an ad lib. diet, fatter male hamsters also have a larger lean body mass (Fig. 3), but, as in bears, fattening among females seems to be almost independent of the growth in other tissues. The rates of expansion of each depot, however, are tightly coordinated, with selective enlargement of the superficial adipose tissue (Pond et al. 1987). These little hamsters are convenient as laboratory animals and their body composition is readily manipulated by factors other than food supply, such as environmental temperature and daylength, and so may be useful as models for investigations of sex differences in growth of lean and fat tissue.

These observations indicate that sex differences in adipose tissue metabolism probably take the form of a large number of minor quantitative contrasts that may be tightly linked to habits and life-history strategy rather than a single major switch. Clearly, much more 


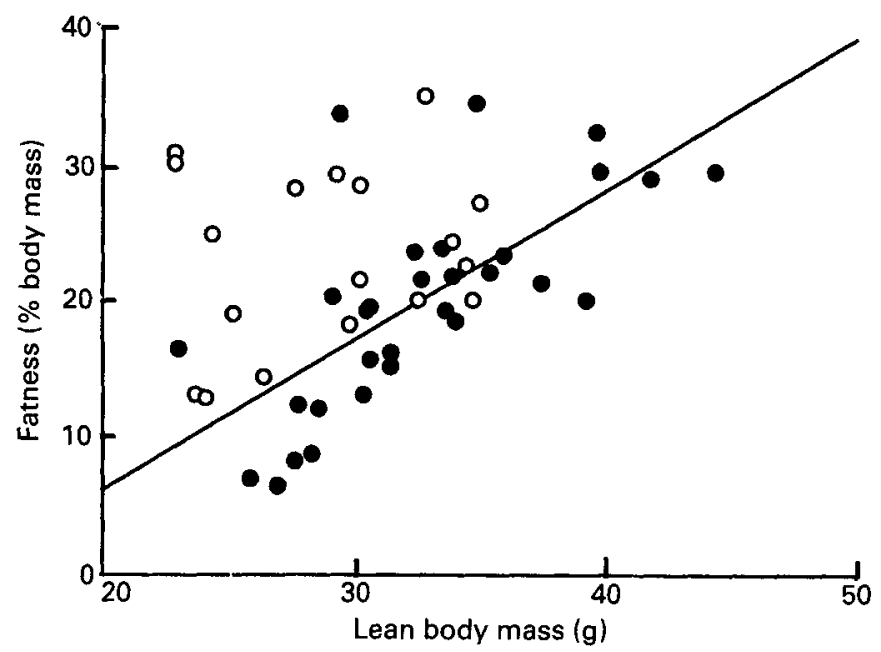

Fig. 3. Sex differences in the relationship between fatness (all dissectible adipose tissue as percentage live body mass) and lean body mass in captive dwarf hamsters (Phodopus sungorus). (O) Adult males ( $n$ 30, $r 0.70$, $P<0 \cdot 01$ ); (O), adult females ( $n 18, r 0 \cdot 18$, not significant). Data adapted from Pond et al. (1987) and Mattacks \& Pond (1988).

must be learned about sex differences in basic adipose tissue metabolism, as well as about the modulating effects of reproductive hormones, before we can hope to understand the full picture. Perhaps questions about the origin of the conspicuous sex differences in humans should be reframed as: how did the sex differences in metabolism get out of kilter in this species so that (almost uniquely) they lead to substantial differences in gross anatomy?

\section{CONCLUDING REMARKS}

Much is now known about the molecular mechanisms of basic adipose tissue function and for mechanisms that control total body composition the spotlight has moved from the lipid-storing and lipid-utilizing tissues to brain and endocrine mechanisms. Impressive though they are, the concepts that emerge from such studies hardly begin to explain why the distribution and anatomical relations of adipose tissue differ greatly between species and to a lesser extent between individuals of the same species, particularly humans. The search for functional and phylogenetic explanations for these features may be among the most important reasons for continuing with the study of adipose tissue per se. A fuller understanding of the functional anatomy of adipose tissue would also help to elucidate sex differences and enable biologists to foresee the long-term consequences of manipulating adipose tissue distribution.

\section{REFERENCES}

Ashwell, M. (1985). Methodological approaches to the study of adipose tissues: their impact on research into the aetiology of obesity. In New Perspectives in Adipose Tissue: Structure, Function and Development, pp. 271-302 [A. Cryer and R. L. R. Van, editors]. London: Butterworths. 
Ashwell, M., McCall, S. A., Cole, T. J. \& Dixon, A. K. (1986). Fat distribution and its metabolic complications: interpretations. In Human Body Composition and Fat Distribution, pp. 227-242. [N. G. Norgan, editor]. Den Haag, Euronut.

Björntorp, P., Karlsson, M. \& Petterson, P. (1982). Expansion of adipose tissue storage capacity in different ages of rat. Metabolism 31, 366-373.

Engfeldt, P. \& Arner, P. (1988). Lipolysis in human adipocytes: effects of cell size, age and regional differences. Hormone and Metabolism Research 19, Suppl., 26-29.

Enzi, G., Gasparo, M., Biondetti, P. R., Fiore, D., Semisa, M. \& Zurlo, F. (1986). Subcutaneous and visceral fat distribution according to sex, age and overweight, evaluated by computed tomography. Journal of Clinical Nutrition 44, 739-746.

Frayn, K. N., Coppack, S. W., Humphreys, S. M. \& Whyte, P. L. (1989). Metabolic characteristics of human adipose tissue in vivo. Clinical Science 76, 509-516.

Garn, S. M., Sullivan, T. V. \& Hawthorne, V. M. (1987). Differential rates of fat change relative to weight change at different body sites. International Journal of Obesity 11, 519-525.

Johnston, F. E., Wadden, T. A., Stunkard, A. J., Peña, M., Wang, J., Pierson, R. N. \& Van Itallie, T. B. (1988). Body fat deposition in adult obese women. I. Patterns of fat distribution. American Journal of Clinical Nutrition 47, 225-228.

Laber-Laird, K., Shively, C. A., Karstaedt, N. \& Bullock, B. C. (1991). Assessment of abdominal fat deposition in female cynomolgus monkeys. International Journal of Obesity 15, 213-220.

Mårin, P., Rebuffé-Scrive, M. \& Björntorp, P. (1990). Uptake of triglyceride fatty acids in adipose tissue in vivo in man. European Journal of Clinical Investigation 20, 158-165.

Mattacks, C. A. \& Pond, C. M. (1988). Site-specific differences in the rates of fatty acid/triacylglycerol substrate cycling in adipose tissue and muscle of sedentary and exercised dwarf hamsters (Phodopus sungorus). International Journal of Obesity 12, 585-597.

Mattacks, C. A. \& Pond, C. M. (1991). Site-specific differences in the uptake in vivo of 2-deoxyglucose by adipose tissue in the Djungarian hamster, Phodopus sungorus. Nutrition Research 11, 1083-1086.

Mattacks, C. A., Sadler, D. \& Pond, C. M. (1987). The effects of exercise on the activities of hexokinase and phosphofructokinase in superficial, intra-abdominal and intermuscular adipose tissue of guinea-pigs. Comparative Biochemistry and Physiology 87B, 533-542.

Newsholme, E. A. (1977). The regulation of intracellular and extracellular fuel supply during sustained exercise. Annals of the New York Academy of Sciences 301, 81-91.

Pond, C. M. (1986). The natural history of adipocytes. Science Progress, Oxford 70, 45-71.

Pond, C. M. (1987). Some conceptual and comparative aspects of body composition measurements. In Techniques in the Behavioral and Neural Sciences. Vol. 1, Feeding and Drinking, pp. 499-529 [F. M. Toates and N. E. Rowland, editors]. Amsterdam: Elsevier Science Publishers.

Pond, C. M. \& Mattacks, C. A. (1987a). Comparative aspects of hexokinase and phosphofructokinase activity in intermuscular adipose tissue. Comparative Biochemistry and Physiology 87B, 543-551.

Pond, C. M. \& Mattacks, C. A. (1987b). The anatomy of adipose tissue in captive Macaca monkeys and its implications for human biology. Folia Primatologia 48, 164-185.

Pond, C. M. \& Mattacks, C. A. (1991). The effects of noradrenaline and insulin on lipolysis in adipocytes isolated from nine different depots of guinea-pigs. International Journal of Obesity 15, 609-618.

Pond, C. M., Mattacks, C. A., Colby, R. H. \& Ramsay, M. A. (1992a). The anatomy, chemical composition and metabolism of adipose tissue in wild polar bears (Ursus maritimus). Canadian Journal of Zoology 70, 326-341.

Pond, C. M., Mattacks, C. A., Colby, R. H. \& Tyler, N. J. (1993). The anatomy, chemical composition and maximum glycolytic capacity of adipose tissue in wild Svalbard reindeer (Rangifer tarandus platyrhynchus) in winter. Journal of Zoology (In the Press).

Pond, C. M., Mattacks, C. A. \& Sadler, D. (1992b). The effects of exercise and feeding on the activity of lipoprotein lipase in nine different adipose depots of guinea-pigs. International Journal of Biochemistry $\mathbf{2 4}$, $1825-1831$.

Pond, C. M., Mattacks, C. A., Thompson, M. C. \& Sadler, D. (1986). The effect of age, dietary restriction, exercise and maternity on the abundance and volume of adipocytes in twelve adipose depots of adult guinea-pigs. British Journal of Nutrition 56, 29-48.

Pond, C. M. \& Ramsay, M. A. (1992). Allometry of the distribution of adipose tissue in Carnivora. Canadian Journal of Zoology 70, 342-347.

Pond, C. M., Sadler, D. \& Mattacks, C. A. (1987). Sex differences in the distribution of adipose tissue in the Djungarian hamster Phodopus sungorus. Nutrition Research 7, 1325-1328. 
Presta, E., Leibel, R. L. \& Hirsch, J. (1990). Regional changes in adrenergic receptor status during hypocaloric intake do not correlate with changes in adipocyte size or body shape. Metabolism 39, 307-315.

Ramsay, M. A., Mattacks, C. A. \& Pond, C. M. (1992). Seasonal changes and sex differences and in the cellular structure and chemical composition of adipose tissue in wild polar bears (Ursus maritimus). Journal of Zoology (In the Press).

Rebuffé-Scrive, M. (1987). Regional adipose tissue metabolism in women during and after reproductive life and in men. Recent Advances in Obesity Research 5, 82-91.

Rössner, S. \& Björntorp, P. (editors) (1989). In Obesity in Europe 88. London: John Libbey.

Sjöström, L. \& Williams-Olsson, T. (1981). Prospective studies on adipose tissue development in man. International Journal of Obesity 5, 597-604. 